\title{
Improving Sustainability of Programmes in Strategic Environmental Assessment Procedures: the QUAlitative Structural Approach for Ranking (QUASAR) the Environmental Effects.
}

\author{
Gaia Galassi*1, François Levarlet ${ }^{1}$
}

\begin{abstract}
Environmental sustainability is grounded on sustainable tools of planning and programming. In turns, environmental sustainability of plans and programs is ensured by Strategic Environmental Assessment. The Strategic Environmental Assessment differs from the Environmental Impact Assessment in that a quantitative environmental assessment of plans or programs is often not possible. A qualitative approach for the assessment of environmental effects and for the ranking of different possible choices and scenarios of sustainability, the QUAlitative Structural Approach for Ranking (QUASAR), is presented as follows, for the first time. This approach is based on the parameterization of the characteristics of each potential interaction between the plan or program and its natural environment.

QUASAR makes it possible to quantify the effects on a previously determined scale of values, making the assessment of the sustainable development paths reproducible and not depending on the evaluator. We described the application of the QUASAR approach to a real case study, the Cross Border cooperation Program Italy - Albania Montenegro 2014-2020, showing it is functioning for a objective and repeatable assessment of effects as well for the comparison of different scenarios of development, based here on different allocation of financial resources.
\end{abstract}

Keywords: Sustainable development; Strategic environmental assessment; Methodology for the assessment of environmental effects

\section{Introduction}

Environmental sustainability is grounded on sustainable tools of planning and programming. One of the main tool for include sustainability concerns in plans and programmes is the Strategic Environmental Assessment (SEA). SEA is a conventional way of formalizing the process of assessing, at the earliest possible stage, the environmental impacts of decisions made at policy, planning, and program levels (Partidario, 1996). According to Pope et al. (2004) the way it can lead to sustainability is twice. First, it contributes to integration of environmental considerations into decisionmaking (see, for example, Sheate et al., 2003); in addition, SEA provides a sound basis to include sustainability concerns (Verheem and Tonk, 2000; Gibson, 2001).

In past, worldwide, SEA has emerged mainly as a tool for the integration of environmental considerations in policies, and it has assumed different forms. For example, in the United States, the preparation of legislative and programmatic 
Environmental Impact Statements has been an integral element of US practice under the National Environmental Protection Act (NEPA) 1969 (Sadler, 2000). Elsewhere, it has taken the form of an extension of the Environmental Impact Assessment (EIA), including public inquiries and environmental reviews (see, e.g. Sadler, 1996; Bregha et al., 1990).

In the European Union the SEA was introduced by the Directive 42/2001/CE. The Directive, which aim is to provide for a high level of protection of the environment and to contribute to the integration of environmental considerations into the preparation and adoption of plans and programs (hereinafter $\mathrm{P} / \mathrm{P}$ ), explicitly states that the strategic assessment has to ensure the inclusion of sustainability principles.

Although the SEA and EIA present several similarities, the concept and the scope are very different. The EIA (see directive 2011/92/EU) is finalized at pointing out possible effects resulting from the realization of projects. Conversely, the SEA has to investigate if and how the provisions included in $\mathrm{P} / \mathrm{P}$ will interact with the environmental objectives stated for the considered area. In other words, SEA has to be carried out earlier than EIA, under conditions that involve less information, higher uncertainty and less concreteness, which is the case with political decisions (Ness et al., 2007). Shifting the attention to a "strategic" level, SEA introduces the advantage of addressing the development at macro-economic level in a sustainable way. On the other hand, the SEA requires the assessment of something that is not yet defined as a project and, in some cases, for which the localization has not even been determined.

In spite of the numerous publications on SEA (see for example Buckley, 1998; Fischer, 2003; Therivel, 2012), there is no an uniform consensus on how to go about applying the assessment of possible effects on the environment. As discussed by Brown and Th'erivel (2000) there are not any available SEA methodologies which are generally applicable to all strategic actions and in all socio-political contexts: it is necessary to select the most appropriate tool, case by case. Nevertheless, P/P can be divided in two main groups corresponding to two different main categories of approach for the assessment:

- $\quad \mathrm{P} / \mathrm{P}$ containing concrete provisions (as land use destination, localization of projects, and so on);

- $\quad \mathrm{P} / \mathrm{P}$ containing strategic provisions (as strategic objectives, targets, driving of development or financial instruments).

While in the first case a quantitative approach to evaluation is possible (with methodologies similar for example to those used for the EIA, often based on GIS Geographic Information System instruments), in the case of strategic provisions the most used approach is the qualitative one. However, the qualitative approach has several limitations. First of all, it does not allow to easily compare different alternatives: for example, with a qualitative approach it is possible to assess if a provision is good or bad in an environmental sense, but not whether one choice is better or worse than another. In addition, a qualitative assessment is often based on a subjective judgment, and can be influenced by the experience of the evaluator.

In this paper for the first time we present the application to a SEA example of a new qualitative approach for the assessment of environmental effects and for the ranking of different possible choices, the QUAlitative Structural Approach for Ranking (QUASAR). 
Through the parameterization to the characteristics of each potential interaction between the $\mathrm{P} / \mathrm{P}$ and its natural-environment, QUASAR allows to "quantify" the effects on a previously determined scale of values. Amongst the advantages of this approach, there are the reproducibility of the evaluation process and the not-dependency of the results from the evaluator.

A similar approach was previously developed by Cristopher Pastakia (Pastakia and Jensen, 1998) for the screening procedure in the EIA. Screening is a preliminary check, carried out before the preparation of a detailed project, to assess if an intervention could have negative environmental effects and, consequently, if the EIA procedure is required. RIAM is a matrix method developed to obtain subjective judgment in a transparent way and it is based on the standard definition of concepts used in the EIA process. With RIAM, different impacts and their significance can be evaluated using commonly defined criteria, each of which has its own ordinal scales and can thus be translated into a numerical form (Ij $\mathrm{I}^{\mathrm{a}}$ s et al., 2010). RIAM has also been applied in SEA procedure (see, for example Li et al., 2014). QUASAR, unlike RIAM, is constructed on P/P structure and for SEA procedure: it is based on the interference between $\mathrm{P} / \mathrm{P}$ provision and environmental objectives and its aim is to rank the contribution to the $\mathrm{P} / \mathrm{P}$ to the environmental sustainability in the context considered.

The paper is organized as follows. In Section 2 the methodological approach is presented; in Section 3 the application of the QUASAR approach to a real case of study is presented; finally, in Section 4 conclusions are drawn.

\section{Methodological approach}

The SEA, as the name itself states, acts at a strategic level. This means that it is necessary to assess the possible effects taking place from the $\mathrm{P} / \mathrm{P}$ with respect to the environmental objectives relevant to the specific $\mathrm{P} / \mathrm{P}$. The aim of this work is not how to point out environmental objectives. Nevertheless, it is a good practice to extract the environmental objectives from the external coherence analysis, concerning the relationship between the $\mathrm{P} / \mathrm{P}$ and the main environmental plans, programs and strategies acting in the interested area.

Once a list of relevant environmental objectives has been drawn up, this will be crossed with each provisions of the $\mathrm{P} / \mathrm{P}$ considered. Contrary to projects, plans and programs do not require a detailed description of actions. Some typologies of $\mathrm{P} / \mathrm{P}$ have a "strategic" nature, and contain objectives and targets for development of specific policies. Broadly speaking, the nature of the $\mathrm{P} / \mathrm{P}$ provisions is heterogeneous and can vary between general actions, activities or objectives, with different degrees of definition; we will refer to them as "elements".

The undefined nature of provisions included in most $\mathrm{P} / \mathrm{P}$, makes the assessment procedure not straightforward. The identification of possible effects has to start by pointing out all the possible interactions between $\mathrm{P} / \mathrm{P}$ elements and each environmental objective previously identified. The first question to answer is then: "could this $P / P$ element interact in any way with the environmental objective?" The possible answers for each environmental objective considered are yes and no. If the answer is yes, another question arises: "is this element (action/objective/ activity) of the $P / P$ contributing or contrasting the achievement 
of this environmental objective?" In this case it would be possible to answer that the $\mathrm{P} / \mathrm{P}$ element contributes to or contrasts with the environmental objective considered. This first part of the analysis can be based on a literature review, on consultation of databases as well, or on direct consultations with experts through a Delphi approach or other instruments of stakeholders participation.

The two questions allow to identify the first two elements for the assessment of environmental effects (see Table 1). What we identify with $i$, is the interaction: $i$ can assume values 0 (no interaction) or 1 (interaction). Assumptions about the magnitude of the effect are not possible at this stage. The second element for the characterization of the effect is its nature, $n$, that can be positive $($ score +1$)$ if the interaction contributes to the achievement of an environmental objectives, or negative (-1) if the interaction represents an obstacle to (i.e. contrasts with) the environmental objective. We note that the RIAM method (Pastakia and Jensen, 1998), uses changes in the current condition to directly assess the magnitude of the effect. We remark the essential difference between EIA and SEA procedures, that makes not possible (or, at least, not ever possible), to "quantify" the contribution (or the obstacle) of a $\mathrm{P} / \mathrm{P}$ provision to a given environmental objective, at an early stage of planning. For this reason, differently from Pastakia and Jensen (1998) and consequent works (see, for example, Ij”as et al., 2010; Kuitunen et al., 2008; El-Naqa, 2005), we can not attribute directly any value of significance to the nature of the effect.

Once the presence and the nature of the interaction has been established, the next step is to assess the magnitude of the possible effect. In order to do this, four characteristics of the effects will be considered: the permanence, the reversibility, the probability and the diffusion. A description of these characteristics is given in the follow. These characteristics straight derive from the SEA Directive 42/2001/CE, Annex II, "Criteria for determining the likely significance of effects". The point 2 of this Annex, on the characteristics of the effects and of the area likely to be affected, asks to consider in particular "the probability, duration, frequency and reversibility of the effects" and the "the magnitude and spatial extent of the effects (geographical area and size of the population likely to be affected)". Some methodological guidance on SEA suggests to use such characteristics to evaluate the environmental effects (see, for example, Environment, 2003; ODPM and WAG, 2012). What is missing, is a generalizable and repeatable approach for using these characteristics (or criteria) in assessing environmental effects in a wide range of plans and programmes. The QUASAR method proposes a definition and a parametrization of these criteria to obtain a ranking in the assessed effects. The numerous tests performed on the application of this method have shown that they can be applied to a wide range of types of $\mathrm{P} / \mathrm{P}$ elements (more generic, as simple objective, or more specific, as clearly defined and localized actions). Each of these variables can assume the value 0 or 1 , as shown in Table 1 . Using only two degree of value allows to reduce the arbitrariety around the choice.

In addition to the characteristics of effects, we have included a parameter to account for particular critical issues, values or emergencies related to the specific environmental topics on which we are evaluating the effect. Similarly to the characteristics above, also this parameter, the value (identified with $v$ ) derives from the SEA Directive 42/2001/CE, Annex II (second to last and last item of point 2). A similar criterion has been used in the 
RIAM method as improved by (Ij $j$ as et al., 2010), which considers "the susceptibility of the target environment". Here, the value allows enhancing the magnitude of the effect for specific situation. It is 1 if no peculiar emergency or criticality has been detected for the environmental topic in the area considered, and it is 1.5 otherwise.

The Magnitude $M$ of the expected effect can be derived from

$M=(i \times n) \times(c 1+c 2+c 3+c 4) \times v$

where the nature $n$, the interaction $i$ and the value $v$ have been described above and the criteria $c 1, \ldots, 4$ can assume values of 0 or 1 , according to their definitions.

Criterion $c 1$ accounts for the permanence of the possible effect: it answers to the question "For how long will the effect manifest itself ?" . The RIAM method (Pastakia and Jensen, 1998), has included a "permanence" criterion to define "whether a condition is temporary or permanent, and should be seen only as a measure of the temporal status of the condition". If applicable (i.e. if $i \neq 0$ ), permanence will be 0 if the effect is temporary, or 1 if the effect is permanent, i.e. if it remains after the end of the elements that generated it.

Criterion $c 2$ is about the reversibility and it assumes value of 0 if the effect is reversible and 1 if it is not. Similarly to the permanence, also the reversibility was included in the RIAM method (Pastakia and Jensen, 1998). The concept of reversibility is not uniformly defined. According to Kuitunen et al. (2008), the reversibility of impact signifies a situation in which the original state will be restored after the activity is finished. In our application, having little information on the characteristics of the alteration of the environment generated by the P/P element, similarly to Kuitunen et al. (2008), we define as "not reversible" those effects that required an intervention (measured in physical, monetary, or other terms) to restore the initial conditions.

Criterion $c 3$ gives information on probability. This is often the most difficult criterion to assess. The concept of probability in the SEA context is also linked to the causality: less known is the relation of cause and effect, more difficult is to relate action proposals with environmental effects, even when a possible interaction has been assessed. According to Perdico'ulis et al. (2007), the causality has not been adequately handled in SEA guidance document. To avoid introducing to much discretion, the criterion has only two degrees of probability: "certain" and "not-certain" . All the effects that surely will be generated by the element considered, will assume a value of 1 . For the others, which are possible but not certain, the value is 0 .

Criterion $c 4$ is about the spatial diffusion of the effect. This importance of effect in terms of spatial extent has been considered in the RIAM method, and especially in its development by Ij as as et al. (2010) (see their criterion A1). In this context, it is referred to the range at which the element can manifest its effect but also accounts for the spatial scale of the $\mathrm{P} / \mathrm{P}$. It will be 0 if the effect is localized (sub regional, less than the total coverage of the $\mathrm{P} / \mathrm{P}$ ), or 1 if it has a widespread diffusion beyond the territorial limits of the area covered by the $\mathrm{P} / \mathrm{Ps}$.

According with the value of Table 1 , the magnitude of expected effects $(M)$ can range between -6 and +6 . To facilitate the communication about the assessment, we can divide the effects of each nature (positive and negative) into three classes, according to Table 2. 


\section{Discussion}

A first application of the method has been experimented on the Cross Border Cooperation Program Italy - Albania - Montenegro 2014 - 2020 (hereinafter CBCP). The case study below is based on the draft of the СВCP used for the consultation with environmental authorities and public during July-August 2015. The Program is organized in four Priority Axis (PA), divided in one or more Specific Objectives (SO). The Program logic of intervention was set considering the template provided by the EUCommission DG Regio. The logic of intervention is similar for all the 60 cross border programs $(\mathrm{CBC})$ falling under the new ETC regulation in force for the entire programming period 2014-2020.

The PA1 is devoted to strengthening the cross-border cooperation and competitiveness of SMEs. It includes only one SO, aimed at strengthening crossborder competitiveness, contributing to cross-border smart specialization strategies. Possible actions from the $\mathrm{SO}$ range from access to research results and technology transfer for SMEs, to networking of Intermediary Organizations (such as Chambers of Commerce), to feasibility studies and pilot actions for innovation.

The PA2 aims for a good governance of natural and cultural heritage for the exploitation of cross border sustainable tourism and territorial attractiveness and it has two SOs. The SO 2.1 contains actions focused on development and branding of macro-regional tourism routes, including the development of common ITC promotional tools in cultural/tourism sector, the creation of products for specific tourism categories and the development of new cross border cultural/tourist routes. Possible actions are also finalized to develop common models for the smart and sustainable tourism management. The SO 2.2 concerns the promotion of cultural heritage through promotion and realization of cultural and creative activities, initiatives and events, with mainly immaterial possible actions.

The PA3 is about environment protection, risk management and low carbon strategy and it is divided in two SOs. The SO3.1 is on cross-border cooperation strategies on water landscapes and it aims at strengthening the management and the cooperation between partners in order to enhance the capacity of relevant actors to improve the water cycle management with attention to coastal and inland environmental risks prevention and biodiversity safeguard. The SO 3.2 is on coordination in implementation of innovative practices and tools on the reduction of carbon emission (also through the promotion of energy efficiency).

The PA4 is on cross border sustainable networks and accessibility. The only SO selected for this PA (SO4.1) is devoted to increasing coordination between relevant stakeholders to promote sustainable cross border connections in the cooperation area. Actions included in the $\mathrm{CBCP}$ are wide spreading, ranging from the cooperation to improve multimodal connections, to the realization of small scale physical infrastructures, to coordination to intercept new traffic flows. The environmental objectives relevant for the Italy-Albania-Montenegro CBCP have been extracted by the external coherence analysis and are listed in Table 3, where the environmental topic and a short id. are also outlined.

The context analysis, together with the external coherence analysis, has allowed to point 
out the environmental issues representing a criticality in the context considered. In the case of the CBCP here considered, the context analysis has shown that climate change and associated risks are critical issues (especially in terms of desertification and coastal erosion) and they represent a key point in strategic policies at different territorial levels. Marine environments and activities are the focus of the CBCP itself: the cooperation has to be finalized to improve the sustainability of activities on shared marine space. In this sense, the "Marine biodiversity and ecosystems" issue is a strength for the cooperation area and it needs of more attention during the assessment process.

In Figure 1, the assessed effects are summarized. The values of magnitude obtained with Eq. 1 are presented according with the symbols in Table 2. The effective magnitude of effects pertaining to the same class is sometime different. For example, for the SO2.1, the not significant negative effects on "pressure on fresh water" (WA2) and on "inland ecosystem conservation" (IB1) have obtained a score of -1 and -2 , respectively. The first effect accounts for the possible increase of pressure on water (in terms of use and of pollution) deriving from an increase of tourism: it is not certain, reversible, and temporary (because linked to the tourist flows): the only criterion activated is the diffusion, since it is expected that it will affect the cross border cooperation area. The possible increase of tourism is also expected to generate a negative effect on inland ecosystem. Like the previous one, this effect is widespread on the cross border cooperation area. In addition, since a deteriorated ecosystem needs time to recover its initial conditions (where this is possible), the "Permanence" criterion has also been activated.

The detail of the magnitude of each of the effects is shown in Figure 2. An overview of this Figure shows that some SOs has a generally positive impact on the environment whereas a negative contribution is expected from others. For example, the SO3.1 could have positive effects on climate change adaptation, by actions for risk management and prevention, cross-border early warning system and procedures for risk assessment. These effects, that are direct and involve a whispered spatial horizon, became significant considering the priority given to climate change adaptation issues in this analysis. In addition, positive indirect effects (not significant) can be expected on inland biodiversity and ecosystem, as consequences of the improved management of coastal and natural area. On the other hand, the increment of transport flows promoted by the SO4.1 should have negative effects (significant, because with a wide spatial diffusion and certain with respect to the foreseen action) on GHG emission, and on air quality and human health (not significant), in terms of emission of, and exposition to, pollutants. The realization of small physical infrastructures has negative effects on biodiversity: these effects are significant because, even if not certain (depending on the location and on project characteristics), they are likely to be not reversible.

A further application of QUASAR is to compare the environmental effects of different scenarios. In the cooperation programs, a key role is played by the allocation of financial resources. Through QUASAR, the assessment of environmental effects can be used to establish the more sustainable repartition of financial resources. In our case study, three different scenarios have been chosen. The Scenario A considers the allocation of resources for Priority Axis chosen for the version of the CBCP used as case study. In the Scenario B, an uniform repartition of financial resources between the PAs was 
considered. Finally, the Scenario C offers a favourable division for the PAs with better environmental performances. The percentage allocation of resources, presented in Table 4, has been used to weigh the effects assessed for each environmental objectives, according with the following equation

$\sum \mathrm{A}\left(\mathrm{me}_{\mathrm{A}} \times \mathrm{pp}_{\mathrm{A}}\right)$

where $A$ is the index associated to the Priority Axis, $\mathrm{pp}_{\mathrm{A}}$ represents the percentage of allocation of resources in a given Scenario, and $\mathrm{me}_{\mathrm{A}}$ is the magnitude of the environmental effect for each PA (for PA with two or more SOs, the magnitude considered is the sum of the single effects). The application of Eq. 2 to the three Scenario in Table 4 allows to obtain a different weight for the effects assessed for each environmental objective, as shown in Figure 3. The comparative assessment shows that the cumulative effects change significantly only if the financial repartition is drastically changed. This suggests that an improvement of environmental performance of the Program could be obtained modifying the actions rather than changing the financial framework. The scenario chosen by the OP (Scenario A) represents a good compromise between positive and negative effects. Even if Scenario C presents better environmental performance (it has been chosen to enhance positive effects and to minimize the negative ones), the A makes a realistic attempt to respond to the socio-economic needs of the cooperation area, whilst at the same time producing good environmental performances.

\section{Conclusions}

The QUASAR approach for ranking the qualitative assessment of environmental effects during SEA procedure has been presented. As shown above, this approach allows to perform a transparent and repeatable assessment, when a quantitative approach is not possible. Such kind of analysis allows to identify at an early stage of the programming process, the key critical issues, and to predispose the possible mitigation measure. In addition, ranking the effects allows to assess the available alternatives, driving the decision making towards a sustainable development. During the SEA process of the CBC Program Italy - Albania - Montenegro used here as case study, the preparation of the Environmental Report and the assessment of environmental effects, have allowed to make suggestions for an improvement of the sustainability of the Program: this, in conjunction with the consultation with public and Environmental Authority, has resulted in a more sustainable final version of the $\mathrm{CBCP}$ with strongly reduced negative effects and increased positive ones.

\section{Acknowledgments}

We thank Claudio Polignano, Managing Authority of the Cross Border Cooperation Program Italia - Albania - Montenegro, for the fruitful collaboration during the SEA procedure. 
Figures and Tables

Table 1: Criteria and weight for the QUASAR approach

\begin{tabular}{|c|c|c|c|}
\hline & Giunis & Valur & Mkmilly \\
\hline i & II uyastien & $\mathbf{w l}$ & Yes:Nes \\
\hline $\mathbf{n}$ & Nilure & $-|y|$ & Nhyentis e / Punilize \\
\hline , & Critizullity & $1 / 1.5$ & Nil uppliuhlu / applinublu \\
\hline 1 & Peiluamerise & $\mathbf{w}$ & 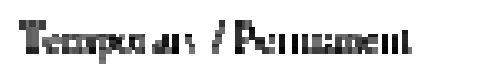 \\
\hline$: 2$ & Randsilily & $\mathbf{w} \mathbf{l}$ & Run arible / Nu Ruminible \\
\hline 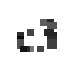 & Pobuhility & $\mathbf{w l}$ & 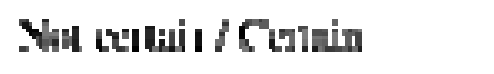 \\
\hline I- & Ditionem & $\mathbf{w}$ & 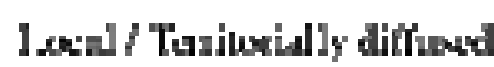 \\
\hline
\end{tabular}

Table 2: Classes for assessment in QUASAR method

\begin{tabular}{|c|c|c|}
\hline 300 & Alsenifotion & Datianion \\
\hline$+4=6$ & 11 & Peinfer nas siptifican difed \\
\hline $2<=4$ & - & 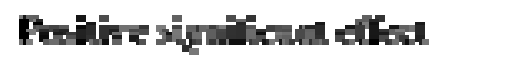 \\
\hline$\tilde{T}<=4$ & $=2$ & 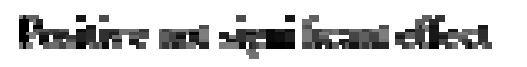 \\
\hline$=0$ & t & Monfled \\
\hline $24=\div$ & ax & 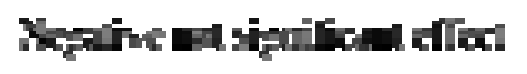 \\
\hline$+4=2$ & - & 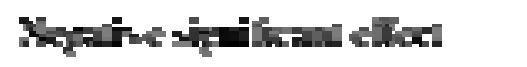 \\
\hline $6<=-4$ & -- & 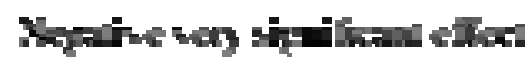 \\
\hline
\end{tabular}


Table 3: Environmental objectives and related topics relevant for the Program, as emerged from the context and the external coherence analysis. The starred $\left(^{*}\right)$ issues are those accounting for peculiar criticality, value or emergencies, and considered for the "value" criterion v in Eq. (1)

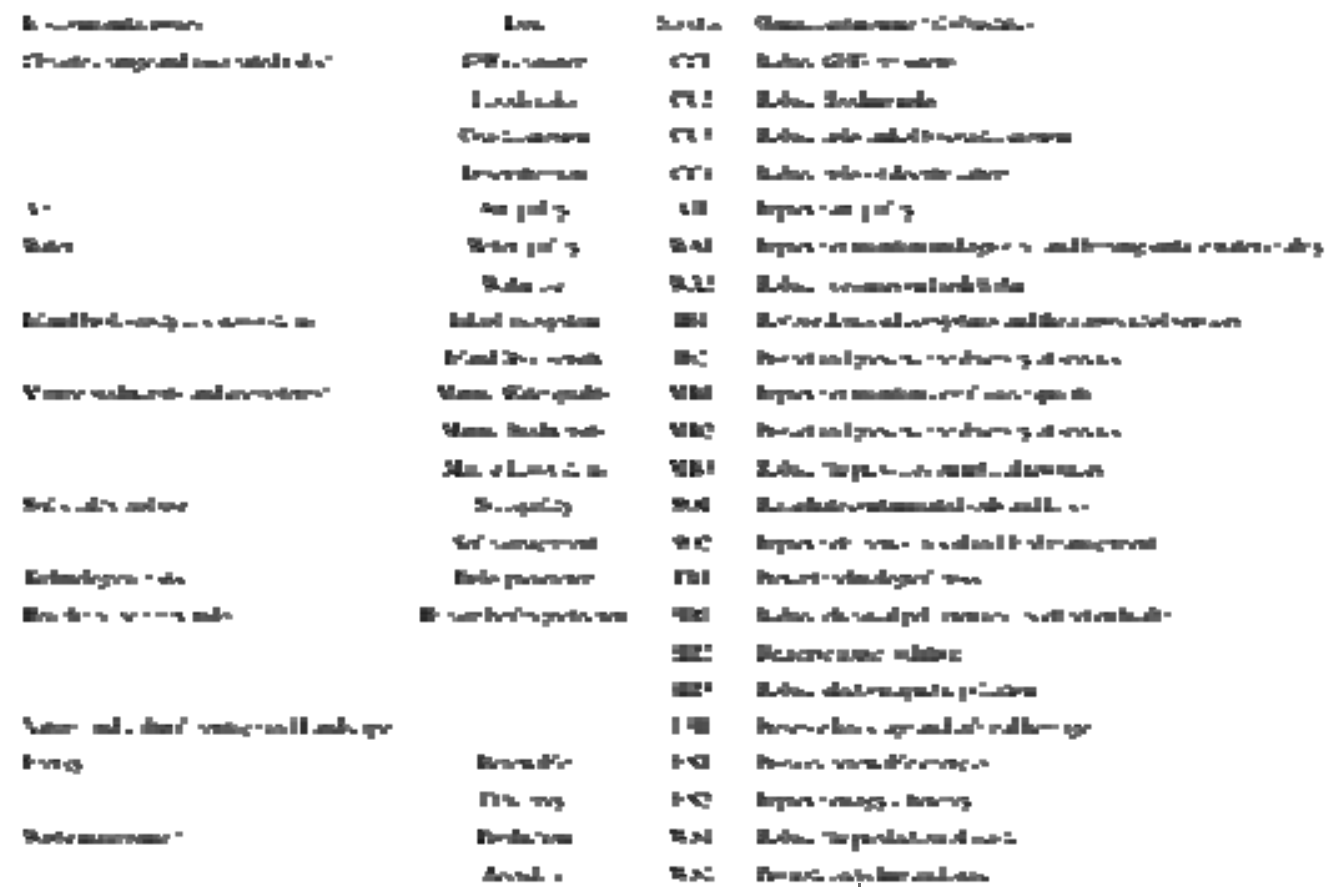




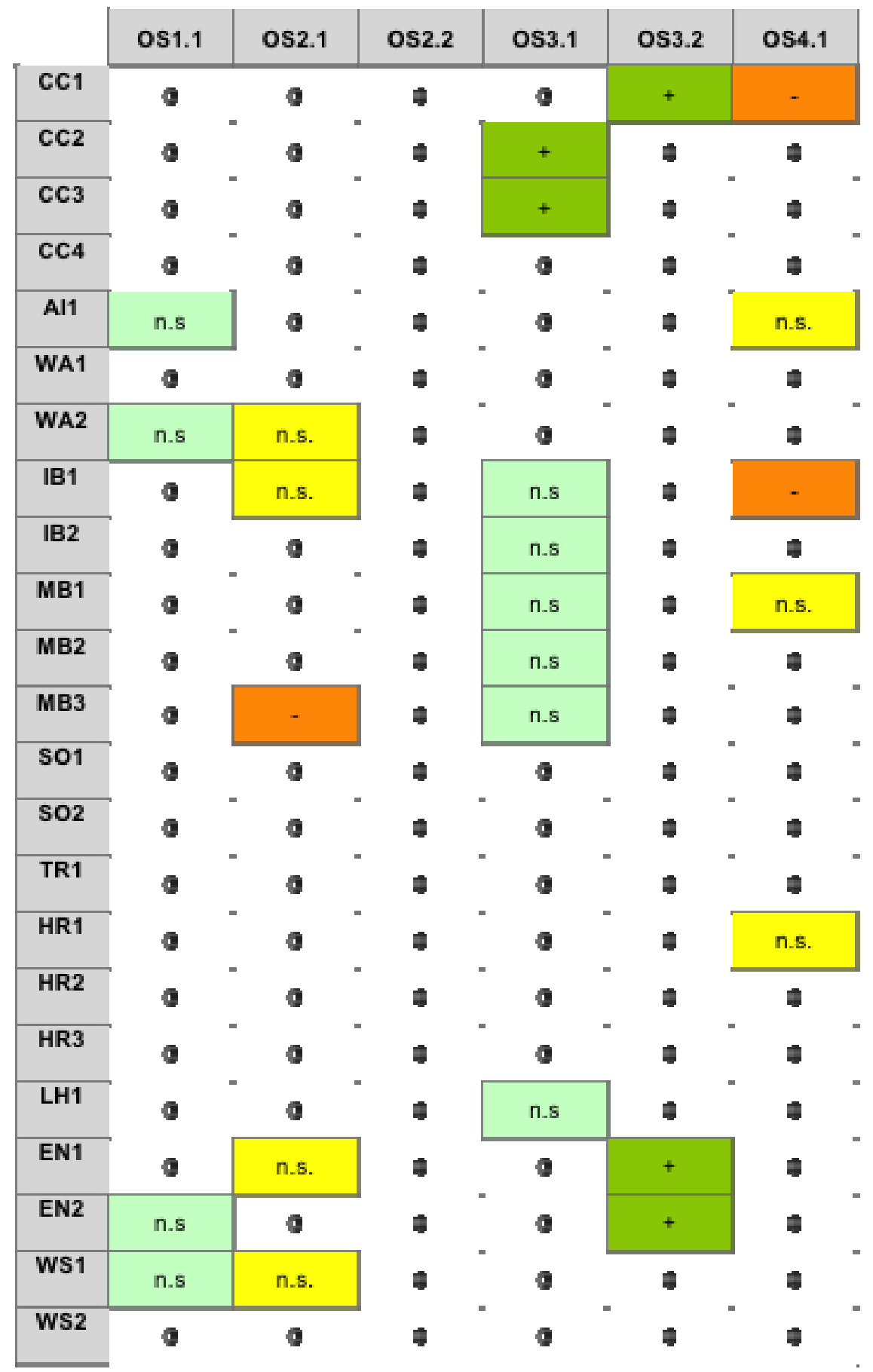

Figure 1: Synthesis of the assessment of possible environmental effects from CBC IT-AL-ME Program. 


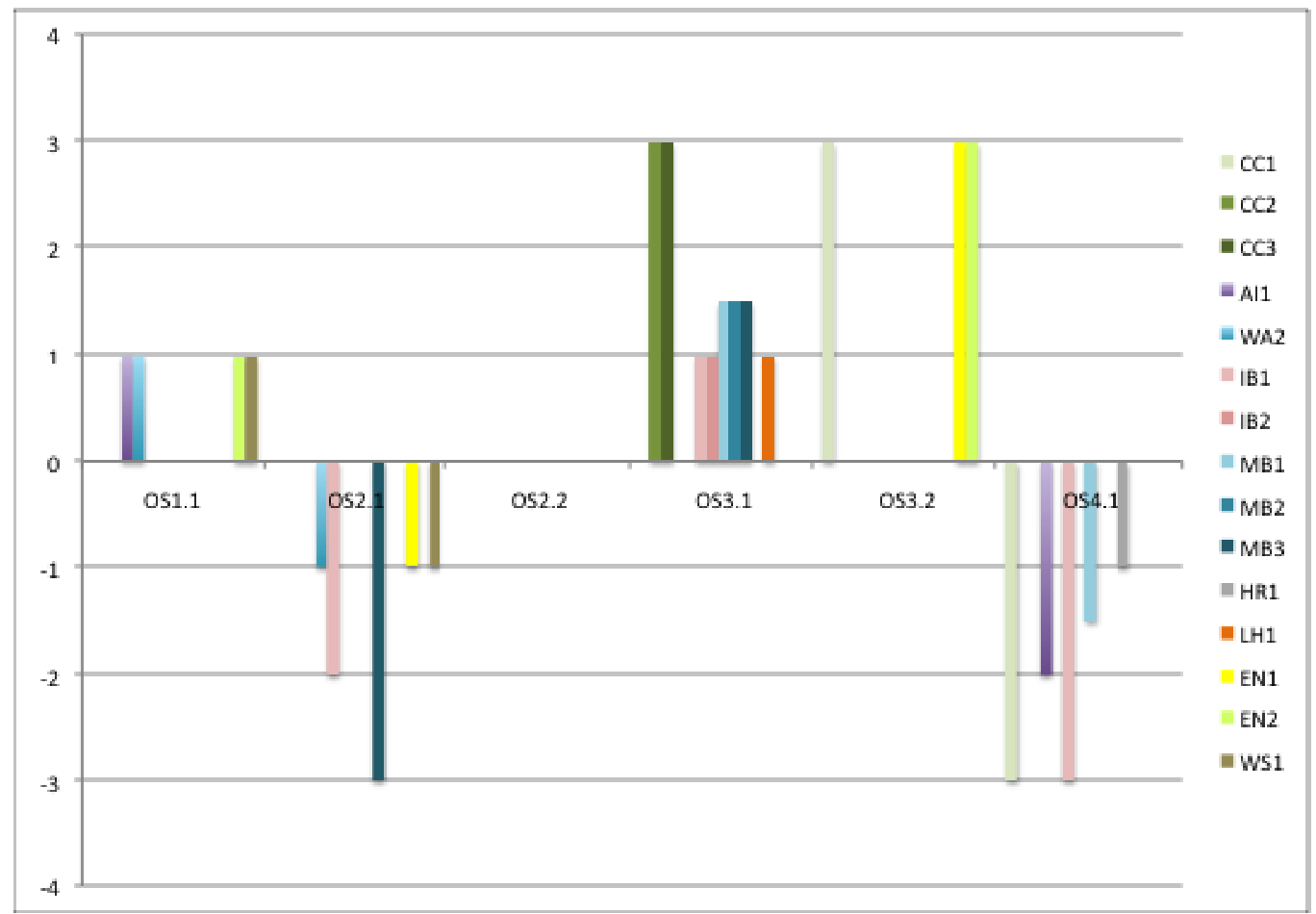

Figure 2: Magnitude of assessed possible environmental effects from each Specific Objectives.

Table 4: Repartition of financial resources of the CBCP for the analysis of scenarios

\section{PAL MA PA PA}

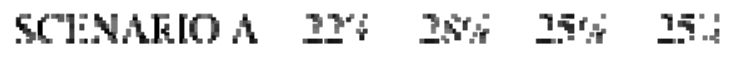

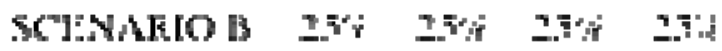

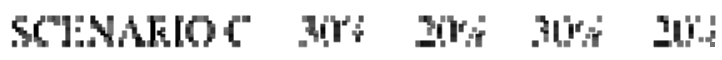




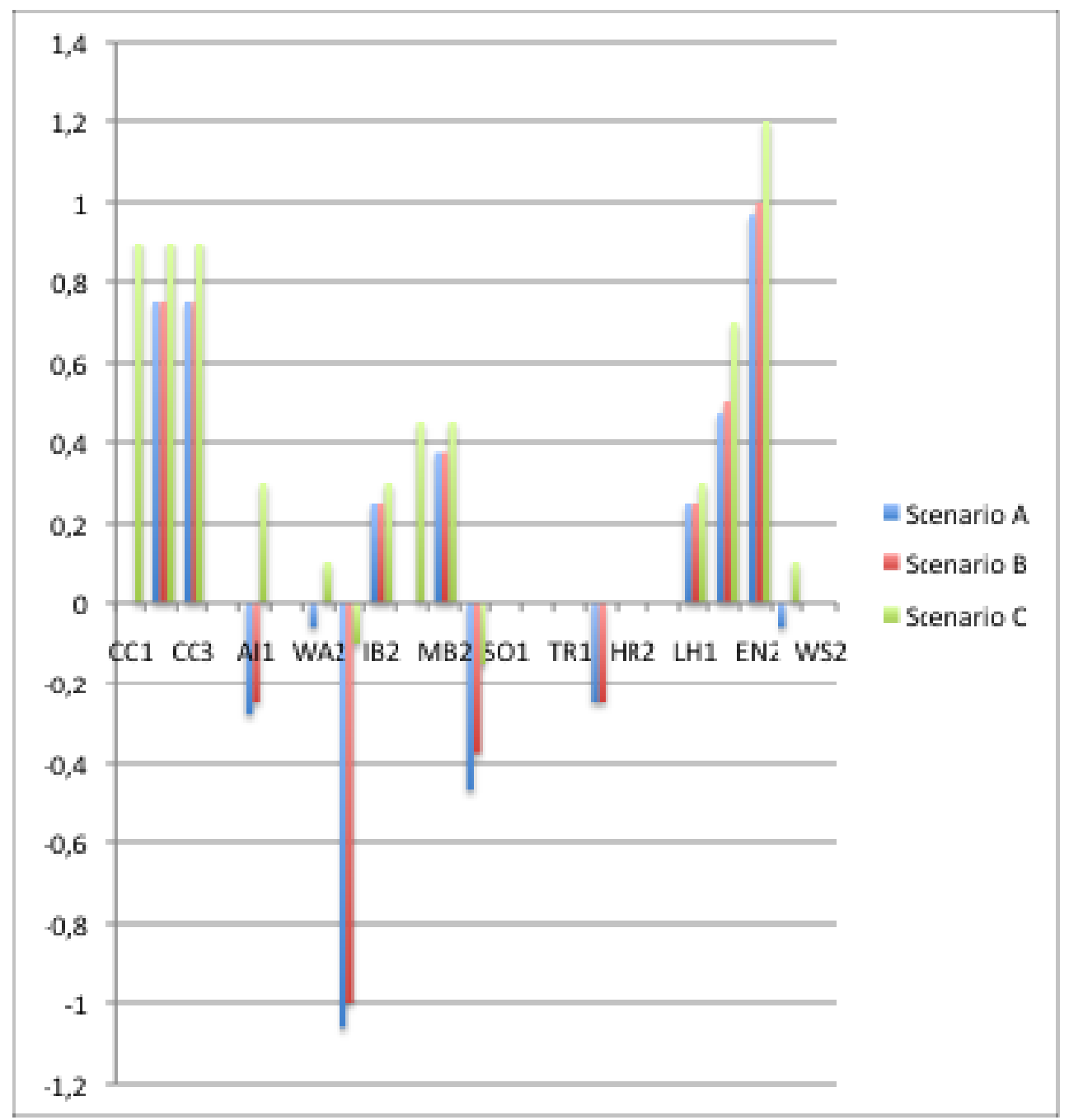

Figure 3: Comparison of environmental effects for different financial scenarios.

\section{References}

Bregha, F., Bendickson, J., Gamble, D., Shillington, T., Weick, E., 1990. The integration of environmental factors in government policy-making. Canadian Environmental Assessment Research Council, Ottawa.

Brown, A., Th'erivel, R., 2000. Principles to guide the development of strategic environmental assessment methodology. Impact Assessment and project appraisal 18 (3), 183-189.

Buckley, R., 1998. Strategic environmental assessment. Environmental Methods Review: Retooling Impact Assessment for the New Century, 77-86.

El-Naqa, A., 2005. Environmental impact assessment using rapid impact assessment matrix (riam) for russeifa landfill, jordan. Environmental Geology 47 (5), 632-639.

Environment, D., 2003. Implementation of Directive 2001/42 on the assessment of the effects of certain plans and programmes on the environment. European Commission. 
Fischer, T. B., 2003. Strategic environmental assessment in post-modern times. Environmental impact assessment review 23 (2), 155-170.

Gibson, R. B., 2001. Specification of sustainability-based environmental assessment decision criteria and implications for determining" significance" in environmental assessment. Ph.D. thesis, University of British Columbia.

Ij”as, A., Kuitunen, M. T., Jalava, K., 2010. Developing the riam method (rapid impact assessment matrix) in the context of impact significance assessment. Environmental Impact Assessment Review 30 (2), 82-89.

Kuitunen, M., Jalava, K., Hirvonen, K., 2008. Testing the usability of the rapid impact assessment matrix (riam) method for comparison of eia and sea results. Environmental Impact Assessment Review 28 (4), 312-320.

Li, W., Xie, Y., Hao, F., 2014. Applying an improved rapid impact assessment matrix method to strategic environmental assessment of urban planning in China. Environmental Impact Assessment Review 46, 13-24.

ODPM, U., WAG, D., 2012. A practical guide to the strategic environmental assessment directive. UK ODPM, London, viewed 28.

Partidario, M. R., 1996. Strategic environmental assessment: key issues emerging from recent practice. Environmental Impact Assessment Review 16 (1), 31-55.

Pastakia, C. M., Jensen, A., 1998. The rapid impact assessment matrix (RIAM) for EIA. Environmental Impact Assessment Review 18 (5), 461-482.

Perdico'ulis, A., Hanusch, M., Kasperidus, H., Weiland, U., 2007. The handling of causality in sea guidance. Environmental Impact Assessment Review 27 (2), 176-187.

Pope, J., Annandale, D., Morrison-Saunders, A., 2004. Conceptualising sustainability assessment. Environmental impact assessment review 24 (6), 595-616.

Sadler, B., 1996. Environmental Assessment in a Changing World. Evaluating Practice to Improve Performance-final Report.

Sadler, B., 2000. Strategic Environmental Assessment: A Rapidly Evolving Approach-7790iied. No. 18. IIED.

Sheate, W. R., Dagg, S., Richardson, J., Aschemann, R., Palerm, J., Steen, U., 2003. Integrating the environment into strategic decision-making: conceptualizing policy sea. European environment 13 (1), 1-18.

Therivel, R., 2012. Strategic environmental assessment in action. Routledge.

Verheem, R., Tonk, J., 2000. Strategic environmental assessment: one concept, multiple forms. Impact Assessment and Project Appraisal 18 (3), 177-182. 